\begin{tabular}{|c|c|}
\hline Title & Transient analy sis of oblique Hanle signals observed in GaA s \\
\hline Author(s) & Lin, Zhichao; Kondo, Kenji; Y amamoto, Masafumi; Uemura, Tetsuya \\
\hline Citation & $\begin{array}{l}\text { Japanese Journal of A pplied Physics (JJA P), 55(4), 04EN03-1-04EN03-5 } \\
\text { https://doi.org/10.7567/JJA P.55.04ENO3 }\end{array}$ \\
\hline Issue Date & 2016-04 \\
\hline Doc URL & http:/hdl .handle.net/2115/64919 \\
\hline Rights & (C) 2016 The Japan Society of A pplied Physics \\
\hline Type & article (author version) \\
\hline File Information & SS15196- library.pdf \\
\hline
\end{tabular}

Instructions for use 


\title{
Transient analysis of oblique Hanle signals observed in GaAs
}

\author{
Zhichao Lin, ${ }^{1}$ Kenji Kondo, ${ }^{2}$ Masafumi Yamamoto, ${ }^{1}$ and Tetsuya Uemura ${ }^{1, *}$ \\ ${ }^{1}$ Division of Electronics for Informatics, Hokkaido University, Sapporo 060-0814, Japan \\ ${ }^{2}$ Research Institute for Electronic Science, Hokkaido University, Sapporo 001-0020, Japan
}

A transient response of nuclear spins in GaAs to a change in a magnetic field was analyzed based on the time evolution of nuclear spin temperature. Simulation results well reproduced our experimental results on transient oblique Hanle signals observed in an allelectrical spin injection device, enabling a quantitative understanding of nuclear spin dynamics in the presence of a hyperfine interaction between nuclei and polarized electrons. Analysis regarding the time evolution of nuclear spin temperature revealed that the hysteretic nature of a nuclear field with respect to the sweep direction of an external field was caused by the delay of time response of nuclear spin temperature to a change in the magnetic field. This analysis provides a deep understanding of nuclear spin dynamics in semiconductors.

*uemura@ist.hokudai.ac.jp 


\section{Introduction}

Spintronics, in which electron spins along with electron charges are used for information processing and memory, is an emerging technology for creating electronic devices with features of nonvolatility, reconfigurable logic functions, and ultralow power consumption, which are called spintronic devices. The understanding of the interplay between electron spins and nuclear spins due to the hyperfine interaction is a major issue in the development of spintronic devices. In the hyperfine interaction, non-equilibrium polarized electrons transmit their spin angular momentum to the lattice nuclei, leading to an effective nuclear spin polarization, referred to as dynamic nuclear polarization (DNP). The polarized nuclear spins will, in turn, affect the electron spins by enhancing electron spin relaxation in semiconductors [1]. Several instances of DNP in semiconductors induced by optical means or electrical means have been reported [2-17]. Furthermore, coherent manipulation of nuclear spins, or Rabi oscillation, which is a key factor for nuclear-spin-based qubits, has been demonstrated electrically in GaAs/AlGaAs quantum Hall systems [18-20] and optically in GaAs/AlGaAs quantum wells [21, 22]. In previous studies, we demonstrated high tunneling magnetoresistance (TMR) ratios of up to $1995 \%$ at $4.2 \mathrm{~K}$ and up to $354 \%$ at $290 \mathrm{~K}$ in magnetic tunnel junctions (MTJs) having Mn-rich $\mathrm{Co}_{2} \mathrm{MnSi}$ electrodes [23], and $2610 \%$ at $4.2 \mathrm{~K}$ and $429 \%$ at $290 \mathrm{~K}$ in $\mathrm{Co}_{2}$ (Mn,Fe)Si MTJs [24]. The obtained high TMR ratios for MTJs with $\mathrm{Mn}$-rich $\mathrm{Co}_{2} \mathrm{MnSi}$ or $\mathrm{Co}_{2}(\mathrm{Mn}, \mathrm{Fe}) \mathrm{Si}$ electrodes are attributed to suppressed $\mathrm{Co}_{\mathrm{Mn}}$ antisites, which leads to the enhanced half-metallicity through a reduced density of minority-spin in-gap states around Fermi level [23-28]. The demonstration of high TMR ratios indicates a high spin polarization of Mn-rich $\mathrm{Co}_{2} \mathrm{MnSi}$ or $\mathrm{Co}_{2}(\mathrm{Mn}, \mathrm{Fe}) \mathrm{Si}$. Thus, these Mn-rich Co-based Heusler alloys are promising spin source materials for a highly efficient spin injection into semiconductors. Indeed, we have demonstrated a high spin injection efficiency from $\mathrm{Co}_{2} \mathrm{MnSi}$ into GaAs via an ultrathin insertion layer of CoFe in an all-electrical spin injection device, resulting in an electron spin polarization of up to $52 \%$ at the ferromagnetic/GaAs interface at $4.2 \mathrm{~K}$ [29]. Furthermore, we have demonstrated Rabi oscillation at a relatively low magnetic field of approximately $0.1 \mathrm{~T}$ in the same device [30].

The polarized nuclear spins affect electron spins as an effective magnetic field or Overhauser field. Thus, one can evaluate the degree of nuclear-spin polarization through the strength of the Overhauser field. To detect the nuclear field, oblique Hanle effect measurements have been widely used [2, 12-17]. While the electrical detection of oblique Hanle signals has been carried out only in a steady state in which the magnetic field was 
swept slowly enough to ensure that the nuclear spin polarization was in equilibrium [14], we investigated the transient response of nuclear spins to a change in the magnetic field along with a time scale needed for DNP through transient oblique Hanle effect measurements in an all-electrical spin injection device with a $\mathrm{Co}_{2} \mathrm{MnSi}$ spin source [17]. However, a rather complicated behavior of nuclear spins against a change in the magnetic field was observed, and only a qualitative understanding for this has been achieved. To fully exploit the nuclear spins in future spintronics, it is necessary to better understand the transient response of nuclear spins. In this paper, we quantitatively analyze the transient response of nuclear spins to a change in the magnetic field in the presence of a hyperfine interaction between nuclei and polarized electrons by using the concept of nuclear spin temperature.

\section{Simulation model}

Nuclear spins are coupled to each other by spin-spin interactions, and also loosely coupled to a lattice by a spin-lattice relaxation mechanism. The time scale for the spin-lattice system to reach thermodynamic equilibrium is characterized by $T_{1}$, which is on the order of $1 \mathrm{~s}$ or more. On the other hand, the interactions inside the nuclear spin system (dipole-dipole interaction of the nuclear magnetic moments) are characterized by a much shorter time, $T_{2}$. Each of the nuclei experiences a fluctuating local field $B_{\mathrm{L}}$ generated by its neighbors. The period of the nuclear spin precession in $B_{\mathrm{L}}$ defines the characteristic time of the interactions inside the nuclear spin system. Owing to these internal interactions, the nuclear spin system reaches the thermodynamic equilibrium internally during a time of order of $T_{2}$, which is given by $1 / \gamma B_{L}$ and is generally on the order of $100 \mu$ s, where $\gamma$ is the gyromagnetic ratio of nuclei. This equilibrium state is characterized by a nuclear spin temperature [31] which in general differs strongly from the lattice temperature because $T_{1}>>T_{2}$. Based on Curie's law, the average nuclear spin $\mathbf{I}_{\mathbf{a v}}{ }^{(\alpha)}$ and resultant Overhauser field $\mathbf{B}_{\mathbf{n}}$ of all nuclei in the presence of an external magnetic field $\mathbf{B}$ are given by [32]

$$
\begin{gathered}
\mathbf{I}_{\mathrm{av}}^{(\alpha)}=\frac{\left(I_{\alpha}+1\right) \mu_{I}^{(\alpha)}}{3 k_{B} \theta_{\alpha}} \mathbf{B}, \\
\mathbf{B}_{n}=\sum_{\alpha} b_{n}^{(\alpha)} \mathbf{I}_{a v}^{(\alpha)} / I_{\alpha},
\end{gathered}
$$

where $\alpha$ denotes the nuclei species, $I_{\alpha}$ is the value of nuclear spin, $\mu_{I}^{(\alpha)}=\gamma_{\alpha} \hbar I_{\alpha}$ is the nuclear magnetic moment, where $\hbar$ is the reduced Planck's constant, $k_{B}$ is the Boltzmann constant, $\theta_{\alpha}$ is the nuclear spin temperature, and $b_{n}{ }^{(\alpha)}$ is the effective nuclear field at $100 \%$ nuclear 
spin polarization. In the case of GaAs, the $b_{n}{ }^{(\alpha)}\left(\alpha={ }^{75} \mathrm{As},{ }^{69} \mathrm{Ga}\right.$, and ${ }^{71} \mathrm{Ga}$ nuclei) values are negative [32].

In the presence of polarized electrons, nuclear spins are effectively polarized through the transfer of spin angular momentum from polarized electrons to nuclei, leading to the cooling of nuclear spin temperature. The sign of the nuclear spin temperature is positive (negative) when the sign of $\mathbf{B} \cdot \mathbf{S}$ is positive (negative), where $\mathbf{S}$ is average electron spin $(|\mathbf{S}|$ $=1 / 2$, corresponding to $100 \%$ polarization). In the case that $\mathbf{B} \cdot \mathbf{S}$ is positive, the spin angular momentum parallel to $\mathbf{B}$ is transferred into the nuclear spin system, leading to an increased nuclear spin polarization. This corresponds to cooling of the nuclear spin temperature below the lattice temperature. For example, for $|\mathbf{S}|=0.25$ and $|\mathbf{B}|=5 \mathrm{mT}$, one finds $\theta_{\alpha} \sim 10^{-6}$ $\mathrm{K}$. When $\mathbf{B} \cdot \mathbf{S}$ is negative, on the other hand, nuclear spins are negatively polarized because a population inversion occurs through the transfer of spin angular momentum antiparallel to $\mathbf{B}$, and the nuclear spin temperature becomes negative (see Fig. 1). However, the picture described above is the one for steady-state nuclear spins. If the external magnetic field is changed faster than the time scale of the DNP, the transient response of nuclear spins should be considered.

Considering the energy balance of the nuclear spin system interacting with polarized electrons, the time $(t)$ evolution of nuclear spin temperature under the DNP process is described by the following equation [32]:

$$
\frac{d}{d t}\left(\frac{1}{\theta_{\alpha}}\right)=-\frac{1}{T_{1 e}}\left(\frac{1}{\theta_{\alpha}}-\frac{1}{\theta_{0 \alpha}}\right),
$$

where $T_{1 e}$ is the characteristic time for the DNP, which can be as long as $100 \mathrm{~s}$ or more, and $\theta_{0 \alpha}$ is the steady-state nuclear spin temperature, which is given by [32]

$$
\frac{1}{k_{B} \theta_{0 \alpha}}=f_{\alpha} \frac{4 I_{\alpha}}{\mu_{I}^{(\alpha)}} \frac{\mathbf{S} \cdot \mathbf{B}}{\mathbf{B}^{2}+\xi B_{L}^{2}},
$$

where $f_{\alpha}(\leq 1)$ is the leakage factor, and $\xi$ is a numerical coefficient defined by the ratio of the nuclear spin polarization rate to the depolarization rate [12]. The steady-state Overhauser field is characterized by $\theta_{0 \alpha}$.

In the electrical oblique Hanle effect measurement using a four-terminal nonlocal geometry in a lateral spin transport device [Fig. 2(a)] [33-35], the Overhauser field can be detected through the detection of the nonlocal voltage $V_{\mathrm{NL}}$ between contacts 3 and 4 . The total magnetic field of $\mathbf{B}+\mathbf{B}_{\mathbf{n}}$ induces Hanle precession for electron spins, resulting in a decrease in the electron spin polarization under detector contact-3. Similarly to the conventional nonlocal Hanle signal, $V_{\mathrm{NL}}$ can be described by [36, 37] 


$$
V_{N L}=A \int_{0}^{\infty} \frac{1}{\sqrt{4 \pi D t}} \exp \left(-\frac{d^{2}}{4 D t}\right) \cos \left(\omega_{L} t\right) \exp \left(-\frac{t}{\tau_{s}}\right) \mathrm{d} t
$$

where $A$ is a constant, $D=l_{\mathrm{sf}}^{2} / \tau_{\mathrm{s}}$ is the diffusion constant, where $l_{\mathrm{sf}}$ is the spin diffusion length and $\tau_{s}$ is the electron spin relaxation time, $d$ is the distance between contact- 2 and contact-3, and $\omega_{L}=g \mu_{\mathrm{B}} B_{\mathrm{z} /} \hbar$ is the Larmor frequency, where $B_{\mathrm{z}}$ is the $z$ component of $\mathbf{B}+$ $\mathbf{B}_{\mathbf{n}}, g$ is an electron g-factor ( $g=-0.44$ for GaAs), and $\mu_{\mathrm{B}}$ is the Bohr magneton.

To simulate a transient oblique Hanle signal, we assumed an oblique magnetic field $\mathbf{B}_{\mathbf{o b}}$, which was applied along the oblique direction with $\varphi=15^{\circ}$ from the $z$-axis in the $x$-z plane - i.e., $\mathbf{B}_{\mathbf{o b}} \equiv B_{\mathrm{ob}}(\mathbf{x} \sin \varphi+\mathbf{z} \cos \varphi)$, where $B_{\mathrm{ob}}$ is the amplitude of the oblique field of $\mathbf{B}_{\mathbf{o b}}$ with both the positive and negative signs, and $\mathbf{x}$ and $\mathbf{z}$ are the unit vectors along the $x$-axis and $z$-axis directions, respectively. The device was first initiated at $B_{\mathrm{ob}}=+42 \mathrm{mT}$ for a hold time ( $t_{\text {hold }}$ ) of $60 \mathrm{~s}$, so that nuclear spins became dynamically polarized. Then $B_{\mathrm{ob}}$ was swept from $+42 \mathrm{mT}$ to $-42 \mathrm{mT}$ (negative sweep direction) and was swept back from -42 $\mathrm{mT}$ to $+42 \mathrm{mT}$ (positive sweep direction) with a sweep rate of $0.18 \mathrm{mT} / \mathrm{s}$. In addition to $\mathbf{B}_{\mathbf{o b}}$, we assumed a constant stray field $B_{s t}=0.5(\mathrm{mT})$ from the ferromagnetic electrodes, which pointed along the $z$-axis direction in the GaAs channel, as part of the external field in our simulation. Then, the total external magnetic field $\mathbf{B}$ was given by

$$
\mathbf{B}=\mathbf{B}_{\mathbf{o b}}+B_{s t} \mathbf{z}=B_{\mathrm{ob}}(\mathbf{x} \sin \varphi+\mathbf{z} \cos \varphi)+B_{s t} \mathbf{z},
$$

where $B_{\mathrm{ob}}$ as a function of $t$ is given by

$$
B_{\mathrm{ob}}(t)= \begin{cases}42 & (0 \leq t \leq 60) \\ 42-0.18(t-60) & \left(60<t \leq 60+\frac{84}{0.18}\right) \\ -42+0.18\left(t-60-\frac{84}{0.18}\right) & \left(60+\frac{84}{0.18}<t \leq 60+\frac{168}{0.18}\right) .\end{cases}
$$

From Eqs. (4), (6), and (7), $\theta_{0 \alpha}$ in Eq. (3) is t-dependent. Thus, we solved Eq. (3) numerically by dividing $t$ into a time step of $1 \mathrm{~s}$, during which $B_{\mathrm{ob}}$ is assumed to be constant. Substituting numerically solved $\theta_{\alpha}$ into Eqs. (1), (2), and (5), we simulated the transient response of $\mathbf{B}_{\mathbf{n}}$ and $V_{\mathrm{NL}}$ against a change in the magnetic field.

Parameters used in our simulation are shown in Table I. The values of $2\left|\mathbf{S}_{\mathbf{0}}\right|, l_{\mathrm{sf}}$, and $\tau_{\mathrm{sf}}$ in Table I were estimated from the fitting of our previous experimental results of the spin-valve signal ( $V_{\mathrm{NL}}$ vs. in-plane magnetic field along the $\mathrm{x}$-axis) and Hanle signals $\left(V_{\mathrm{NL}}\right.$ vs. out-of-plane magnetic field) observed in a lateral spin transport device with $\mathrm{Co}_{2} \mathrm{MnSi} / \mathrm{CoFe}$ electrodes [17], where $\left|\mathbf{S}_{\mathbf{0}}\right|$ is the average electron spin at $\mathbf{B}=\mathbf{0}$. These 
results are shown in Figs. 2(b) and 2(c), respectively, and the details of the device structure and experimental conditions are described in Ref. 17. The projection of $\mathbf{S}$ to $\mathbf{B}$ in Eq. (4) was estimated from $\left|\mathbf{S}_{\mathbf{0}}\right|$. Although the Hanle precession through $\mathbf{B}+\mathbf{B}_{\mathbf{n}}$ changes the component of $\mathbf{S}$ perpendicular to $\mathbf{B}$, it does not change the component of $\mathbf{S}$ parallel to $\mathbf{B}$. Thus, we assumed that $\mathbf{S} \cdot \mathbf{B}$ is equal to $\mathbf{S}_{\mathbf{0}} \cdot \mathbf{B}$ in the simulation. We considered three kinds of nuclei ${ }^{69} \mathrm{Ga},{ }^{71} \mathrm{Ga}$, and ${ }^{75} \mathrm{As}$ in the GaAs channel. The values of $b_{n}{ }^{(\alpha)}, T_{1 e}$, and $f_{\alpha}$ in Table I were treated as fitting parameters. The values of $I_{\alpha}$ and $\gamma_{\alpha}$ were taken from Ref. 38, and the value of $\sqrt{\xi} B_{L}$ was taken from Ref. 14 .

\section{Results and discussion}

Fig. 3 shows (a) a typical oblique Hanle signal observed in $\mathrm{GaAs}$ with a $\mathrm{Co}_{2} \mathrm{MnSi}$ spin source [17] and (b) a corresponding simulation result obtained using Eqs. (1)-(7). Compared with the simulated steady-state signal described in Ref. 17 , we experimentally observed the following two features: (1) An additional side peak was observed at $B_{\mathrm{ob}}<0$ in the negative sweep direction, and (2) no side peak was observed in the positive sweep direction, showing a clear hysteretic nature depending on the sweep direction. These features were well reproduced by using the concept of nuclear spin temperature. The value $T_{1 e}=400 \mathrm{~s}$ used in the simulation is reasonable as a time scale needed for the DNP $[12,13,17]$. These results validate the simulation model used in this study. A slight deviation between the experiment and simulation for $B_{\mathrm{ob}}>0$ is possibly due to the instability of the background signal during the measurement. Although we subtracted a background signal consisting of a constant term and linear and quadratic terms of $B_{\mathrm{ob}}$, some uncertain background signals might be remained.

Now we will discuss the transient behavior of nuclear spins based on the time evolution of nuclear spin temperature. Because $b_{n}{ }^{(\alpha)}$ is negative in GaAs, we know from Eqs. (1) and (2) that if $\theta_{\alpha}$ is positive (negative), $\mathbf{I}_{\mathbf{a v}}$ is parallel (antiparallel) to $\mathbf{B}$, while $\mathbf{B}_{\mathbf{n}}$ is antiparallel (parallel) to $\mathbf{B}$. Thus, a condition that $\mathbf{B}$ and $\mathbf{B}_{\mathbf{n}}$ cancel each other exists only for $\theta_{\alpha}>0$. Fig. 4 shows (a) $B_{\mathrm{ob}}, B_{\mathrm{n}}$ and (b) $1 / \theta_{\alpha}, 1 / \theta_{0 \alpha}$ of ${ }^{75}$ As nuclei as a function of time, and (c) a simulation result of an oblique Hanle signal, and Fig. 5 shows corresponding nuclear spin distribution of the transient oblique Hanle effect measurement. The value of $1 / \theta_{\alpha}$ increased during a holding time due to DNP, resulting in the nuclear field $\mathbf{B}_{\mathbf{n}}$ being generated antiparallel to $\mathbf{B}$ at $t=t_{\text {hold }}$ [state-1 and Fig. 5(a)]. When $B_{\text {ob }}$ reached $27 \mathrm{mT}$ in the negative sweep direction, $\mathbf{B}_{\mathbf{n}}$ and $\mathbf{B}$ cancelled each other out, leading to one side peak in the nonlocal voltage $V_{\mathrm{NL}}$ [state-2 and Fig. 5(a)]. When $\mathbf{B}$ was reversed, $\theta_{0 \alpha}$ became negative 
because $\mathbf{B} \cdot \mathbf{S}<0$. This means that $\mathbf{B}$ and $\mathbf{B}_{\mathbf{n}}$ should be parallel and no cancellation should occur in the steady state. However, we observed a clear side peak at $B_{\mathrm{ob}}=-11 \mathrm{mT}$ [state-3 and Fig. 5(b)], indicating cancellation between $\mathbf{B}$ and $\mathbf{B}_{\mathbf{n}}$. This is because $\mathbf{B}_{\mathbf{o b}}$ was changed before the nuclear spin system reached the steady-state. Indeed, $1 / \theta_{\alpha}$ was delayed with respect to $1 / \theta_{0 \alpha}$ as shown in Fig. 4(b), and $\theta_{\alpha}$ stayed positive for a certain time after $\mathbf{B}$ was reversed. This time delay happened because the external magnetic field was changed much faster than $T_{1}$ and $T_{1 e}$ processes. After $\mathbf{B}$ was reversed, as shown in Fig. 5(b), the nuclear spin system adiabatically changed their spin direction to keep the Zeeman energy in the low-energy state for the negative $B_{\mathrm{ob}}$ as for the positive $B_{\mathrm{ob}}$, resulting in a positive nuclear spin temperature. However, after sufficient time, that is, at $t>405 \mathrm{~s}$, a population inversion of nuclear spins occurred owing to the DNP process and $\theta_{\alpha}$ became negative, indicating $\mathbf{B}_{\mathbf{n}}$ was parallel to $\mathbf{B}$ [state-4 and Fig. 5(c)]. Similarly in the positive sweep direction, $\theta_{\alpha}$ was negative ( $\mathbf{B}$ and $\mathbf{B}_{\mathrm{n}}$ were parallel) at $B_{\mathrm{ob}}<0$, and it stayed negative for a certain time after $\mathbf{B}_{\mathrm{ob}}$ was reversed from the negative direction to the positive direction [state-5 and Fig. 5(d)]. Thus, no cancellation occurred between $\mathbf{B}$ and $\mathbf{B}_{\mathbf{n}}$, resulting in the disappearance of the satellite peak at $B_{\mathrm{ob}}>0$ for the positive sweep direction.

\section{Conclusion}

A transient response of nuclear spins in GaAs to a change in the magnetic field was analyzed by using the concept of nuclear spin temperature. The hysteretic nature of nuclear field with respect to the sweep direction of the external magnetic field, which originated from the transient response of nuclear spins, was well reproduced by this method. From the analysis, we found that this behavior can be understood by the delay of the time response of nuclear spin temperature to a change in the magnetic field. This finding contributes to a fuller understanding of nuclear spin dynamics.

\section{Acknowledgement}

This work was partly supported by JSPS KAKENHI Grant Numbers 25286039 and $15 \mathrm{~K} 13960$. 


\section{References}

[1] M. I. Dyakonov, in Spin Physics in Semiconductors, ed. M. I. Dyakonov (Springer, Heidelberg, 2008), Chap. 11.

[2] M. I. Dyakonov, V. I. Perel, V. L. Berkovits, and V. I. Safarov, Sov. Phys. JETP 40, 950 (1975).

[3] D. Paget, G. Lampel, B. Sapoval, and V. I. Safarov, Phys. Rev. B 15, 5780 (1977).

[4] G. Lampel, Phys. Rev. Lett. 20, 491 (1968).

[5] J. Strand, B. D. Schultz, A. F. Isakovic, C. J. Palmstrøm, and P. A. Crowell, Phys. Rev. Lett. 91, 036602 (2003).

[6] D. Gammon, S. W. Brown, E. S. Snow, T. A. Kennedy, D. S. Katzer, and D. Park, Science 277, 85 (1997).

[7] G. Salis, D. T. Fuchs, J. M. Kikkawa, and D. D. Awschalom, Phys. Rev. Lett. 86, 2677 (2001).

[8] T. Machida, T. Yamazaki, and S. Komiyama, Appl. Phys. Lett. 804178 (2002).

[9] K. Hashimoto, K. Muraki, T. Saku, and Y. Hirayama, Phys. Rev. Lett. 88, 176601 (2002).

[10] M. Poggio, G.M. Steeves, R. C. Myers, Y. Kato, A. C. Gossard, and D. D. Awschalom, Phys. Rev. Lett. 91, 207602 (2003).

[11] K. Ono and S. Tarucha, Phys. Rev. Lett. 92, 256803 (2004).

[12] J. Strand, X. Lou, C. Adelmann, B. D. Schultz, A. F. Isakovic, C. J. Palmstrøm, and P. A. Crowell, Phys. Rev. B 72, 155308 (2005).

[13] P. Van Dorpe, W. Van Roy, J. De Boeck, and G. Borghs, Phys. Rev. B 72, 035315 (2005).

[14] M. K. Chan, Q. O. Hu, J. Zhang, T. Kondo, C. J. Palmstrøm, and P. A. Crowell, Phys. Rev. B 80, 161206(R) (2009).

[15] G. Salis, A. Fuhrer, and S. F. Alvarado, Phys. Rev. B 80, 115332(R) (2009).

[16] J. Shiogai, M. Ciorga, M. Utz, D. Schuh, T. Arakawa, M. Kohda, K. Kobayashi, T. Ono, W. Wegscheider, D. Weiss, and J. Nitta, Appl. Phys. Lett. 101, 212402 (2012).

[17] T. Akiho, J. Shan, H. -x. Liu, K. -i. Matsuda, M. Yamamoto, and T. Uemura, Phys. Rev. B. 87, 235205 (2013).

[18] T. Machida, T. Yamazaki, K. Ikushima, and S. Komiyama, Appl. Phys. Lett. 82, 409 (2003).

[19] G. Yusa, K. Muraki, K. Takashina, K. Hashimoto, and Y. Hirayama, Nature 434, 1001 (2005). 
[20] H. Takahashi, M. Kawamura, S. Masubuchi, K. Hamaya, T. Machida, Y. Hashimoto, and S. Katsumoto, Appl. Phys. Lett. 91, 092120 (2007).

[21] H. Sanada, Y. Kondo, S. Matsuzaka, K. Morita, C.Y. Hu, Y. Ohno, and H. Ohno, Phys. Rev. Lett. 96, 067602 (2006).

[22] Y. Kondo, M. Ono, S. Matsuzaka, K. Morita, H. Sanada, Y. Ohno, and H. Ohno, Phys. Rev. Lett. 101, 207601 (2008).

[23] H. -x. Liu, Y. Honda, T. Taira, K. -i. Matsuda, M. Arita, T. Uemura, and M. Yamamoto, Appl. Phys. Lett. 101, 132418 (2012).

[24] H. -x. Liu, T. Kawami, K. Moges, T. Uemura, M. Yamamoto, F. Shi, and P. M. Voyles, J. Phys. D: Appl. Phys. 48, 164001 (2015).

[25] M. Yamamoto, T. Ishikawa, T. Taira, G. -f. Li, K. -i. Matsuda, and T. Uemura, J. Phys.: Condens. Matter 22, 164212 (2010).

[26] G. -f. Li, Y. Honda, H. -x. Liu, K. -i. Matsuda, M. Arita, T. Uemura, M. Yamamoto, Y. Miura, M. Shirai, T. Saito, F. Shi, and P. M. Voyles, Phys. Rev. B 89, 014428 (2014).

[27] J. -P. Wüstenberg, R. Fetzer, M. Aeschlimann, M. Cinchetti, J. Minár, Jürgen Braun, H. Ebert, T. Ishikawa, T. Uemura, and M. Yamamoto, Phys. Rev. B 85, 064407 (2012).

[28] V. R. Singh, V. K. Verma, K. Ishigami, G. Shibata, T. Kadono, A. Fujimori, D. Asakura, T. Koide, Y. Miura, M. Shirai, G. -f. Li, T. Taira, and M. Yamamoto, Phys. Rev. B 86, 144412 (2012).

[29] Y. Ebina, T. Akiho, H. -x. Liu, M. Yamamoto, and T. Uemura, Appl. Phys. Lett. 104, 172405 (2014).

[30] T. Uemura, T. Akiho, Y. Ebina, and M. Yamamoto, Phys. Rev. B. 91, 140410(R) (2015).

[31] A. Abragam and W. G. Proctor, Phys. Rev. 109, 1441 (1958).

[32] M. I. Dyakonov and V. I. Perel, in Optical Orientation, ed. F. Meier and B. P. Zakharchenya (North-Holland, Amsterdam, 1984), Chap. 2.

[33] X. Lou, C. Adelmann, S. A. Crooker, E. S. Garlid, J. Zhang, S. M. Reddy, S. D. Flexner, C. J. Palmstrøm, and P. A. Crowell, Nat. Phys. 3, 197 (2007).

[34] G. Salis, A. Fuhrer, R. R. Schlittler, L. Gross, and S. F. Alvarado, Phys. Rev. B 81, 205323 (2010).

[35] M. Ciorga, A. Einwanger, U. Wurstbauer, D. Schuh, W. Wegscheider, and D. Weiss, Phys. Rev. B 79, 165321 (2009).

[36] J. Fabian, A. Matos-Abiague, C. Ertler, P. Stano, and I. I. Zutic, Acta Phys. Slov. 57, 565 (2007). 
[37] I. Zutic, J. Fabian, and S. D. Samra, Rev. Mod. Phys. 76, 323 (2004).

[38] D. Paget and V. L. Berkovits, in Optical Orientation, ed. F. Meier and B. P. Zakharchenya (North-Holland, Amsterdam, 1984), Chap. 9. 


\section{Figure Captions}

Fig. 1. (Color online) Schematic images of nuclear spin distribution (a) under an external magnetic field, (b) under the DNP process when $\mathbf{B} \cdot \mathbf{S}>0$, and (c) under the DNP process when $\mathbf{B} \cdot \mathbf{S}<0$, where $\mathbf{S}$ is average electron spin.

Fig. 2. (Color online) (a) Schematic structure of a four-terminal nonlocal device and circuit configuration for oblique Hanle effect measurements. (b) Spin-valve signal and (c) Hanle signals observed in $\mathrm{GaAs}$ with a $\mathrm{Co}_{2} \mathrm{MnSi}$ spin source. Reprinted with permission from Ref. 17. Copyright 2013, American Physical Society.

Fig. 3. (Color online) (a) Oblique Hanle signal observed in GaAs with a $\mathrm{Co}_{2} \mathrm{MnSi}$ spin source. Reprinted with permission from Ref. 17. Copyright 2013, American Physical Society. (b) A corresponding simulation result obtained using Eqs. (1)-(7).

Fig. 4. (Color online) (a) Time evolution of (a) $B_{\mathrm{ob}}$ and $B_{\mathrm{n}}$, (b) $1 / \theta_{\alpha}$ and $1 / \theta_{0 \alpha}$ of ${ }^{75} \mathrm{As}$ isotope in GaAs. (c) A simulation result of oblique Hanle signal.

Fig. 5. (Color online) Schematic images of nuclear spin distribution of the transient oblique Hanle effect measurement for (a) state-1 and state-2, (b) state-3, (c) state-4, and (d) state-5. 
Table I. Summary of simulation parameters.

\begin{tabular}{ll}
\hline \multicolumn{1}{c}{ Parameter } & \multicolumn{1}{c}{ Value } \\
\hline $2\left|\mathbf{S}_{\mathbf{0}}\right|(\%)$ & 4.4 \\
$\tau_{s}(\mathrm{~ns})$ & 10 \\
$l_{\mathrm{sf}}(\mu \mathrm{m})$ & 5 \\
$b_{n}$ of ${ }^{75} \mathrm{As}(\mathrm{T})$ & -3.59 \\
$b_{n}$ of ${ }^{69} \mathrm{Ga}(\mathrm{T})$ & -2.19 \\
$b_{n}$ of ${ }^{71} \mathrm{Ga}(\mathrm{T})$ & -1.99 \\
$T_{1 e}(\mathrm{~s})$ & 400 \\
$f_{\alpha}$ & 0.85 \\
$I_{\alpha}{ }^{\mathrm{a})}$ & $3 / 2$ \\
$\gamma_{\alpha}$ of ${ }^{75} \mathrm{As}^{\mathrm{a})}[\mathrm{rad} /(\mathrm{T} \cdot \mathrm{s})]$ & $4.581 \times 10^{7}$ \\
$\gamma_{\alpha}$ of ${ }^{69} \mathrm{Ga}^{\mathrm{a})}[\mathrm{rad} /(\mathrm{T} \cdot \mathrm{s})]$ & $6.420 \times 10^{7}$ \\
$\gamma_{\alpha}$ of ${ }^{71} \mathrm{Ga}{ }^{\mathrm{a})}[\mathrm{rad} /(\mathrm{T} \cdot \mathrm{s})]$ & $8.158 \times 10^{7}$ \\
$\sqrt{\xi} B_{L}{ }^{\mathrm{b})}(\mathrm{mT})$ & 5 \\
$d(\mu \mathrm{m})$ & 0.5 \\
$B_{s t}(\mathrm{mT})$ & 0.5 \\
\hline
\end{tabular}

a) Ref. $38 . \quad$ b) Ref. 14. 
(a)

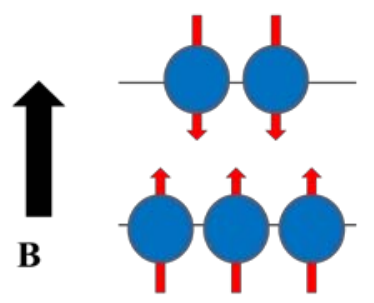

(b)
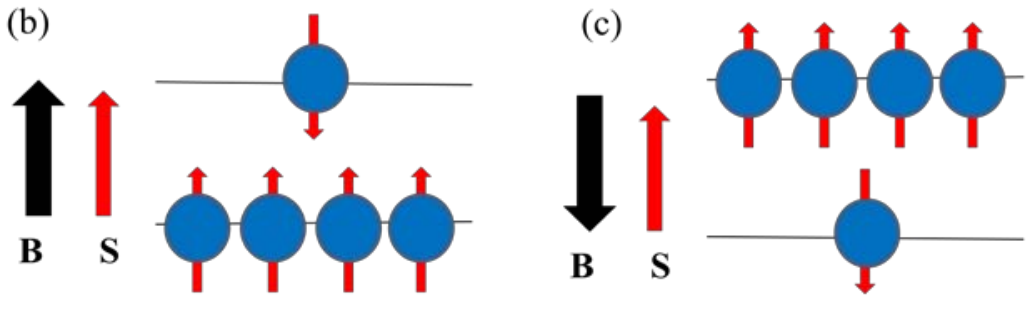

$\mathbf{B} \cdot \mathbf{S}>0, \theta_{\alpha}>0$

$\mathbf{B} \cdot \mathbf{S}<0, \theta_{\alpha}<0$

Fig. 1. (Color online) Schematic images of nuclear spin distribution (a) under an external magnetic field, (b) under the DNP process when $\mathbf{B} \cdot \mathbf{S}>0$, and (c) under the DNP process when $\mathbf{B} \cdot \mathbf{S}<0$, where $\mathbf{S}$ is average electron spin. 

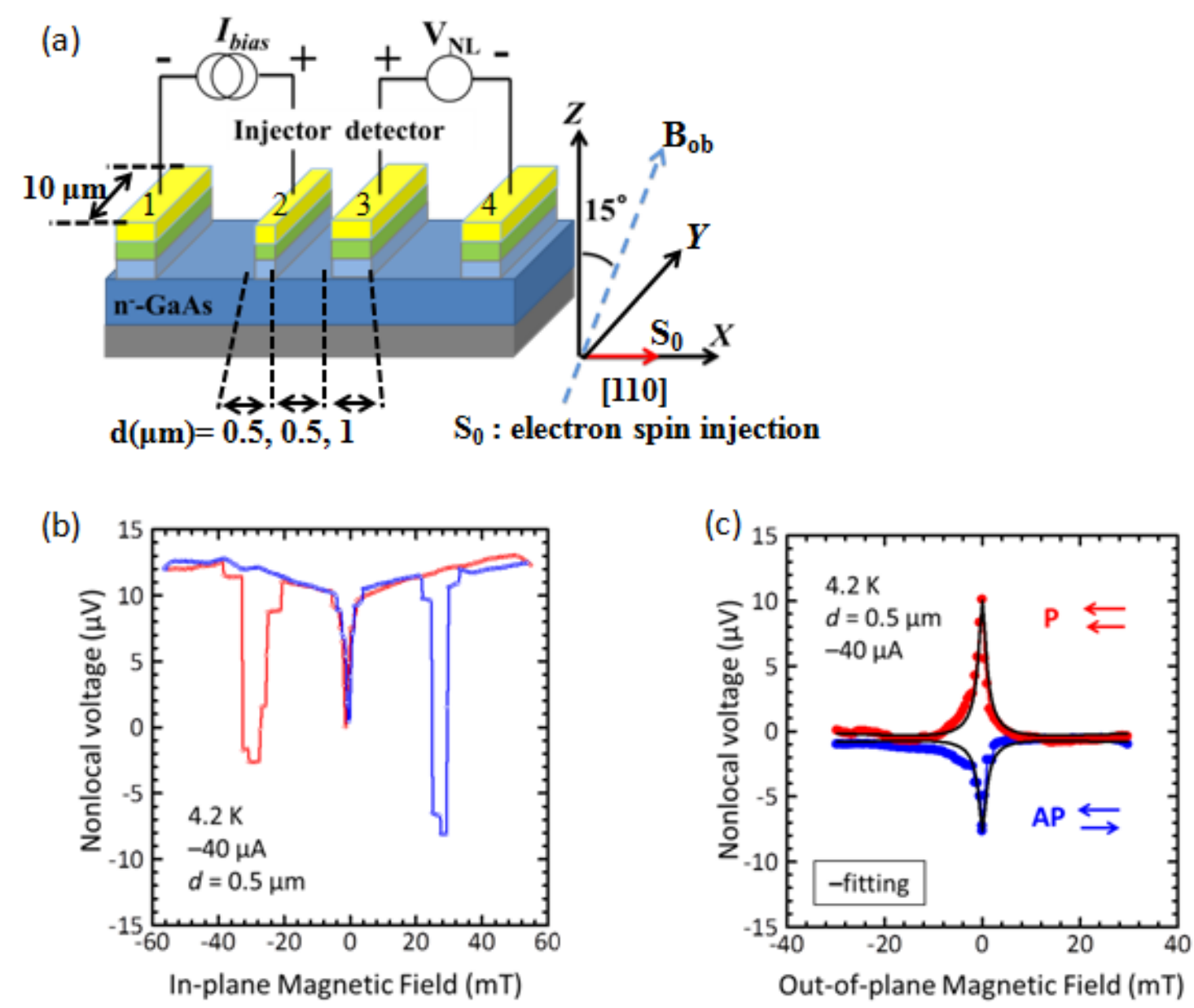

Fig. 2. (Color online) (a) Schematic structure of a four-terminal nonlocal device and circuit configuration for oblique Hanle effect measurements. (b) Spin-valve signal and (c) Hanle signals observed in $\mathrm{GaAs}$ with a $\mathrm{Co}_{2} \mathrm{MnSi}$ spin source. Reprinted with permission from Ref. 17. Copyright 2013, American Physical Society. 

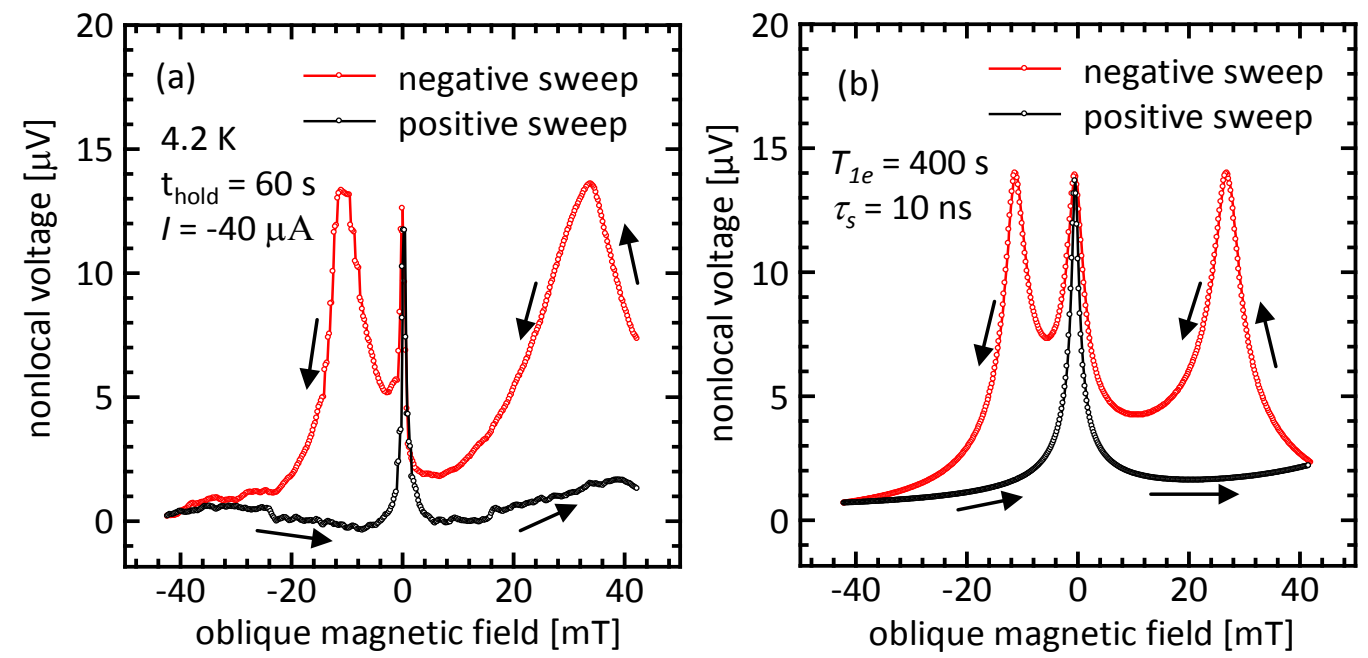

Fig. 3. (Color online) (a) Oblique Hanle signal observed in $\mathrm{GaAs}$ with a $\mathrm{Co}_{2} \mathrm{MnSi}$ spin source. Reprinted with permission from Ref. 17. Copyright 2013, American Physical Society. (b) A corresponding simulation result obtained using Eqs. (1)-(7). 

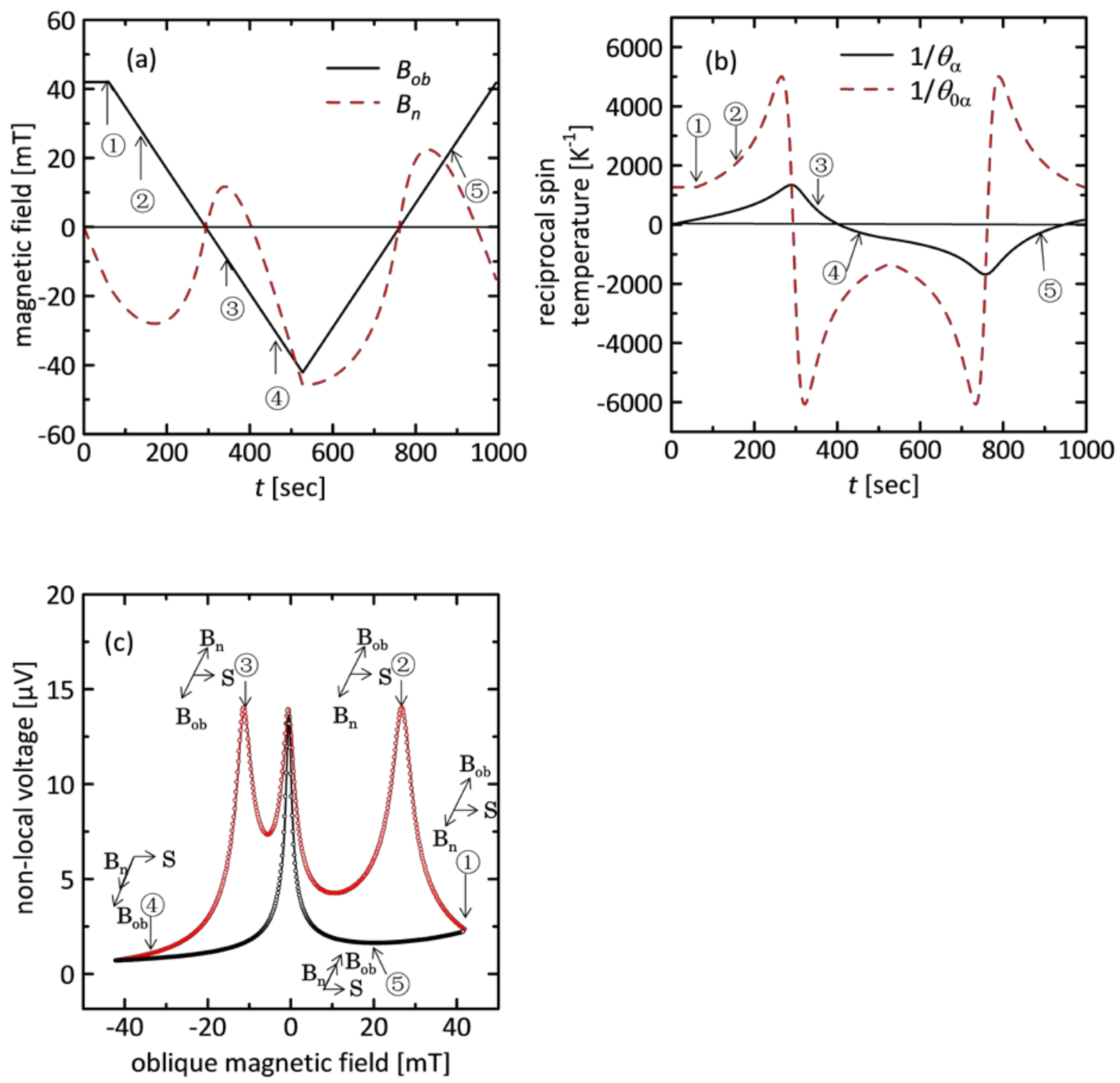

Fig. 4. (Color online) (a) Time evolution of (a) $B_{\mathrm{ob}}$ and $B_{\mathrm{n}}$, (b) $1 / \theta_{\alpha}$ and $1 / \theta_{0 \alpha}$ of ${ }^{75} \mathrm{As}$ isotope in GaAs. (c) A simulation result of oblique Hanle signal. 


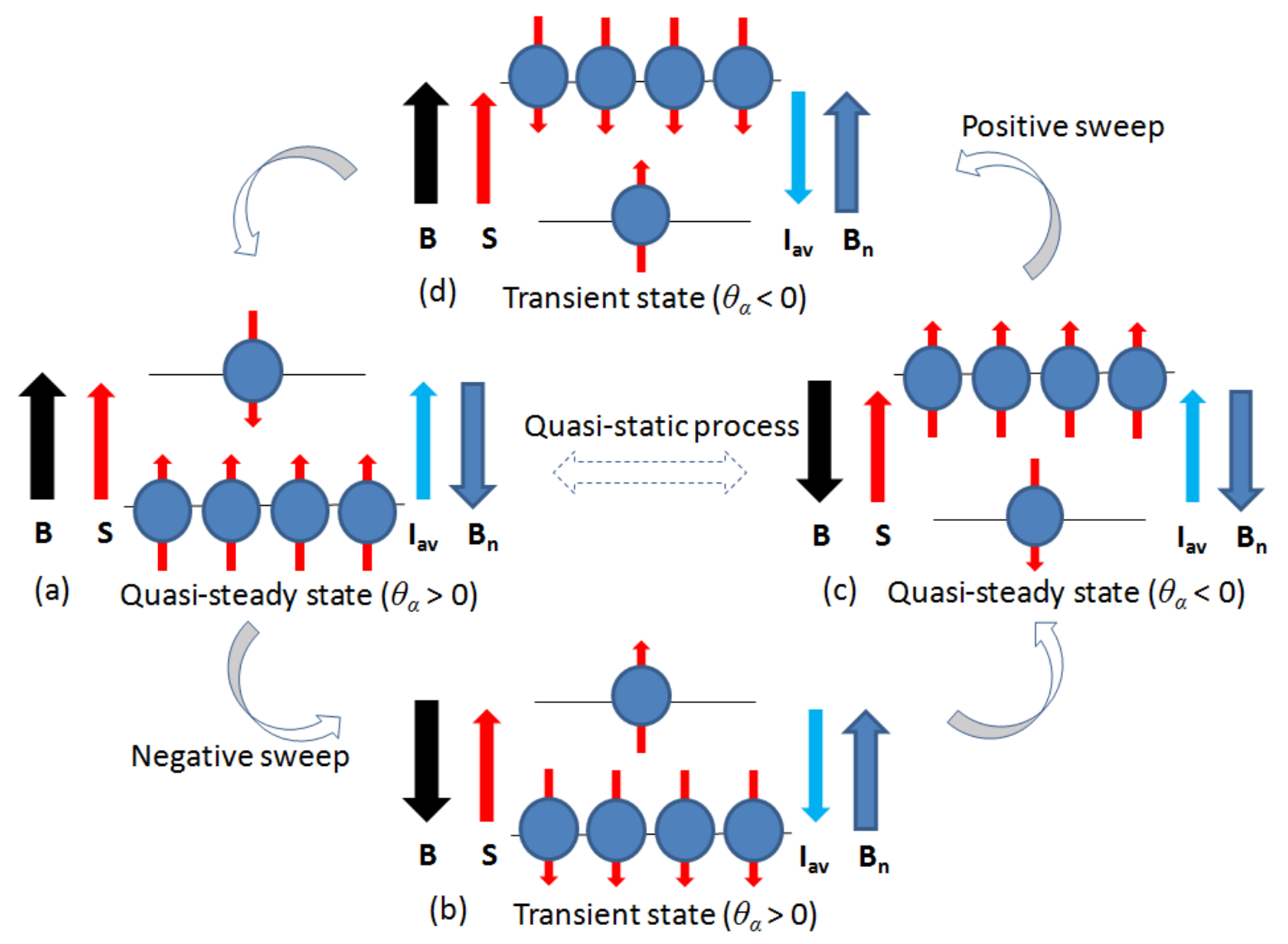

Fig. 5. (Color online) Schematic images of nuclear spin distribution of the transient oblique Hanle effect measurement for (a) state-1 and state-2, (b) state-3, (c) state-4, and (d) state-5. 